\section{Experimental and theoreti- cal characterization of optical signal out-coupling through V-grooved optical fiber cladding}

Seihyoung Lee, Shinyoung Yoon, Jong Jin Lee, Chong Hee Yu, and Hyun Seo Kang

Electronics and Telecommunications Research Institute, Optical Communications Research Center, 1110-6 Oryong-dong, Buk-gu, Gwangju, Korea 500-480

E-mail: seihyoung@etri.re.kr

Abstract. We demonstrate a highly efficient in-fiber outcoupling device. The core mode is coupled to the cladding by a tilted fiber Bragg grating and then the cladding mode is out-coupled from the optical fiber through V-grooved cladding. The light emitting characteristics are investigated experimentally and a maximum out-coupling efficiency of $54.8 \%$ is obtained. (c) 2006 Society of Photo-Optical Instrumentation Engineers.

[DOI: $10.1117 / 1.2402120]$

Subject terms: fiber optic applications; optical devices; optical communications; optical receivers.

Paper 060552LRR received Jul. 13, 2006; revised manuscript received Nov. 3, 2006; accepted for publication Nov. 3, 2006; published online Dec. 4, 2006.

\section{Introduction}

Optical fiber Bragg gratings (FBGs) have been used for optical communication and sensing systems because of their unique wavelength selectivity as well as their compact, flexible, and cost-effective properties. FBGs are usually fabricated in the core region and make it possible to realize fiber in-line devices. In addition, tilted FBGs (TFBGs) are good for sensing the power of the core mode because they can couple the core mode to the cladding mode and/or radiation mode. By directly coupling the core mode to the radiation mode, fiber in-line types of spectrometers $^{1-3}$ and monitors ${ }^{4}$ have been developed, which have a coupling efficiency of mostly $0.1 \sim 3 \%$. Because of the low efficiency, they are not suitable for highsensitivity optical receivers.

In this work, we present a new method that can be applied to the development of new devices for optical communications. Instead of utilizing the radiation mode, the cladding mode is used in this application. In this case, determining how to out-couple the cladding mode from the fiber is a matter of great concern. In consideration of this, the cladding is etched to efficiently out-couple the cladding mode. In our device, two kinds of coupling processes occur. One is the strong core-to-cladding mode coupling by TFBGs and the other is the highly efficient out-coupling of the cladding mode through the V-grooved cladding. Usually, the core-to-cladding mode coupling creates an undesirable

0091-3286/2006/\$22.00 @ 2006 SPIE

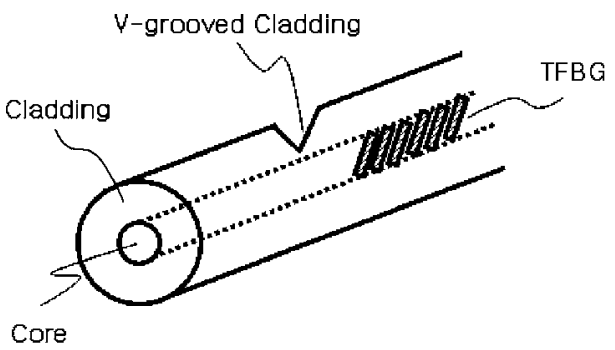

(a)

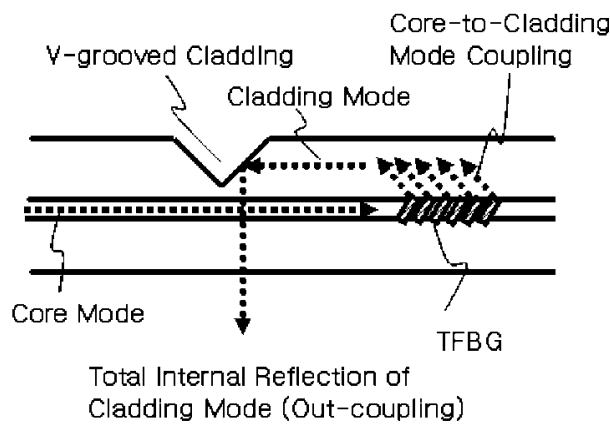

(b)

Fig. 1 (a) Schematic appearance of the device and (b) overall mode coupling process.

crosstalk effect, which results in degradation of the optical communication systems ${ }^{5}$; however, this coupling is necessary to our applications. ${ }^{6}$

\section{Theory}

The rigorous theoretical analysis of the core-to-cladding mode coupling by TFBGs has been well explained in previous studies. ${ }^{7-10}$ The coupling between the modes is maximized if one of the following phase-matching conditions is satisfied:

$2 n_{01}^{c o} \Lambda / \cos \theta=\lambda_{\text {co-co }}$,

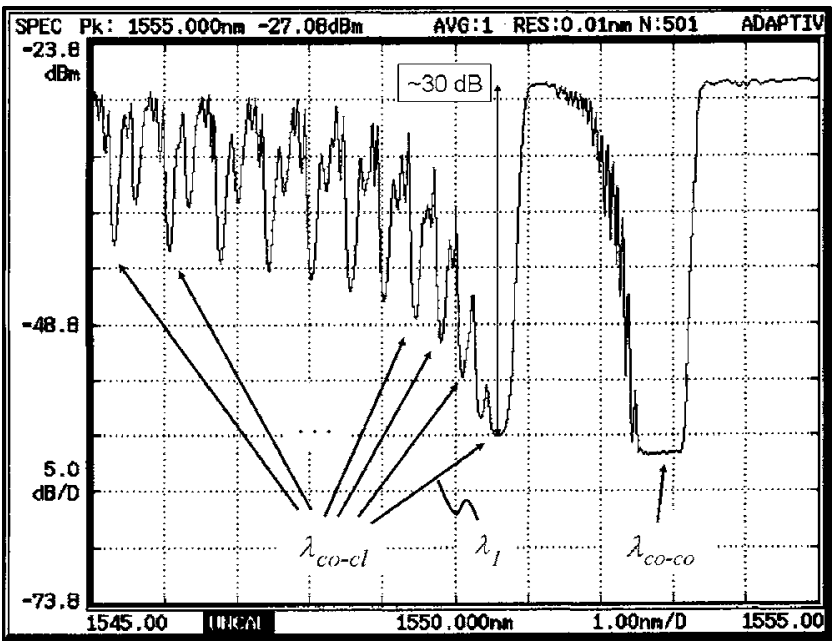

Fig. 2 Transmission spectrum of the fabricated TFBG. 


\section{OE LETTERS}

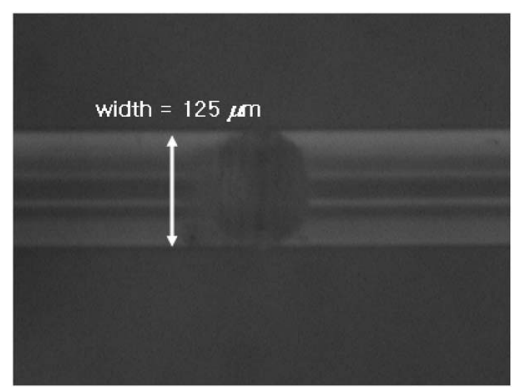

(a)

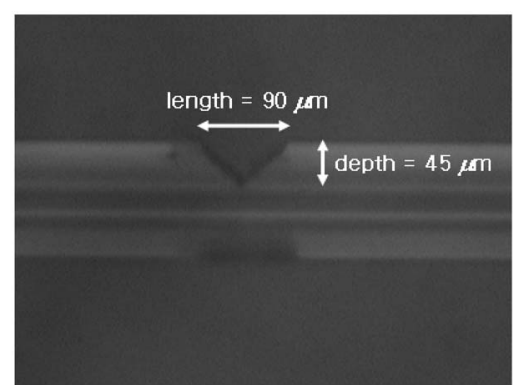

(b)

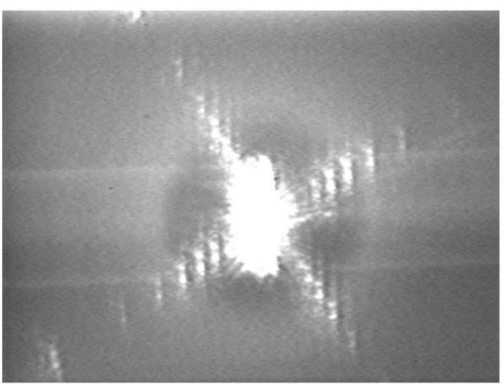

(c)

Fig. 3 Fabricated V-grooved cladding by the femtosecond laser: (a) top view, (b) side view, and (c) infrared camera view of the light of the out-coupled cladding mode at $\lambda=1549.13 \mathrm{~nm}$.

$\left(n_{01}^{c o}+n_{l m}^{c l}\right) \Lambda / \cos \theta=\lambda_{c o-c l}$

where $n_{01}^{c o}$ and $n_{l m}^{c l}$ are effective indices of the fundamental core mode and the $(l m)$-cladding mode, respectively, $l$ and $m$ are the azimuthal and radial mode number, respectively, $\Lambda$ is the untilted grating period, $\lambda_{c o-c o}$ and $\lambda_{c o-c l}$ are resonance wavelengths for the core-to-core mode coupling and core-to- $(\mathrm{lm})$-cladding mode coupling, respectively, and $\theta$ is an actual grating tilt angle. Once the effective indices are calculated by mode analysis, the proper $\theta$ can be calculated from Eq. (2) to induce the core-to- $(l m)$-cladding mode coupling.

The device structure and the overall mode coupling process are depicted in Fig. 1 and the two kinds of coupling are well described in this figure. Importantly, by the effect of total internal reflection (TIR), the cladding mode is outcoupled through the V-grooved cladding.

As mentioned before, TFBGs are used for core-tocladding mode coupling. Therefore, the tilt angle $\theta$ is adjusted to maximize the coupling efficiency at $\lambda_{c o-c l}$ instead of $\lambda_{\text {co-co }}$ so that most power of the core mode is coupled to the cladding mode. The TFBG is fabricated in a hydrogenloaded photosensitive fiber (PS1250/1500, Fibercore Ltd., Southhampton, UK) using a $\mathrm{KrF}$ excimer laser. The tilt angle of the phase mask is set to $2 \mathrm{deg}$, which corresponds to $\theta \sim 2.97 \mathrm{deg}$ for the actual grating tilt angle. ${ }^{11}$ Figure 2 shows the transmission spectrum of the TFBG right after fabrication. As shown in this figure, the maximum core-tocladding mode coupling occurs at $\lambda_{1}=1548.46 \mathrm{~nm}$ and the coupling ratio is about $30 \mathrm{~dB}(\sim 99.9 \%)$. Figure 3 shows the fabricated $\mathrm{V}$-grooved cladding and the light-emitting characteristic. The cladding is cut out by the femtosecond laser. A portion of the cladding can be precisely detached by appropriately focusing the high-power laser beam. The size of the V-groove is about 125 (width) $\times 90$ (length) $\times 45$ (depth) $\mu \mathrm{m}^{3}$.

Once the core mode is coupled to the cladding mode, it propagates in the cladding region and is tightly guided by the air-cladding boundary. Subsequently the cladding mode meets the V-grooved cladding on the way to propagation. Since the refractive index of the air is lower than that of the cladding, TIR occurs as long as the light incident angle to the V-groove is higher than the critical angle. At the operating wavelength, the refractive index of the cladding is about 1.44. As a result, the critical angle is about $44 \mathrm{deg}$. Since the angle of the V-grooved cladding is $45 \mathrm{deg}$, the TIR is satisfied and the most of the cladding mode can be out-coupled to the detector as shown in Fig. 3(c). Theoretically, about half of the cladding mode can be out-coupled since the V-groove can reflect only in one side of the cladding mode as shown in Fig. 1(b).

\section{Experiment}

In our experiment, we measured the output photocurrent of an avalanche photodiode (APD, 124NLVT Cyoptics, Brein-

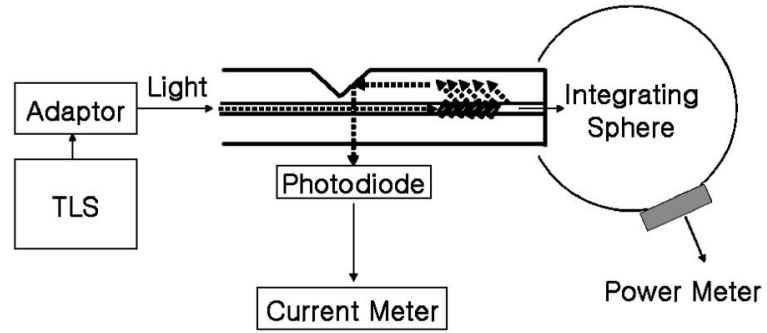

Fig. 4 Measurement setup. The APD is used to detect the light of the out-coupled cladding mode and the integrating sphere is used for the transmitted power. 


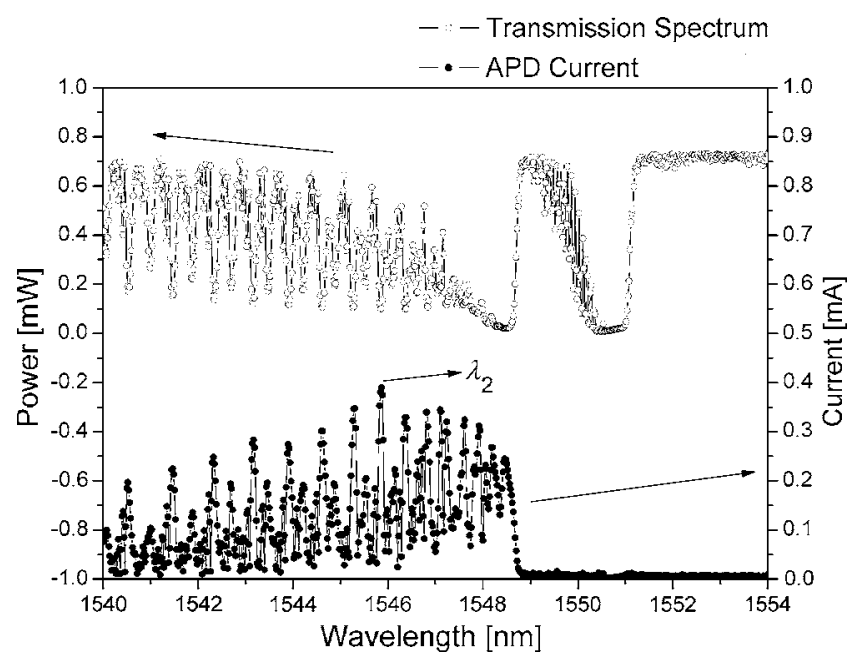

Fig. 5 Measured data. The solid line is the photocurrent of the APD and the dotted line is the transmission spectrum.

igsville, PA) and the transmitted power with respect to the wavelength, and then we calculated the maximum coupling efficiency of the device. Figure 4 briefly shows the measurement setup. Using the tunable laser source, the wavelength is swept from 1540 to $1554 \mathrm{~nm}$ by increments of $0.02 \mathrm{~nm}$ while the power is maintained at $1 \mathrm{~mW}$. On the other hand, the integrating sphere is placed near the end of the fiber to measure the transmission spectrum of the TFBG. Figure 5 shows the measured data. The solid and open circles are photocurrents of the APD and transmitted power, respectively. Since there is no core-cladding mode coupling in the longer wavelength region, there is no photocurrent evidently. The peak wavelength discrepancy of the transmission spectrum between Figs. 2 and 5 is due to the grating stabilization after fabrication. There are local maxima values in the photocurrent and definitely those occur at the wavelengths where the core-to-cladding mode coupling is strong. On the other hand, the maximum photocurrent is measured at $\lambda_{2}=1545.86 \mathrm{~nm}$ but the maximum core-to-cladding mode coupling occurred at a different wavelength, i.e., $\lambda_{1}$. This is because, in our guess, the outcoupling efficiency depends on whether the core mode is coupled to a lower or higher cladding mode. In this point of view, the core mode seems to be coupled to the high-order and low-order cladding mode for $\lambda_{1}$ and $\lambda_{2}$, respectively.

Because of the adaptor loss, the actual power launched into the device is different from the tunable laser source source power and is estimated to be about $0.73 \mathrm{~mW} \mathrm{ob-}$ tained from the transmission spectrum in Fig. 5. The maximum photocurrent is about $0.4 \mathrm{~mA}$ and corresponds to $0.4 \mathrm{~mW}$, in which the multiplying factor $M=9$ is applied. Therefore, in consideration of $M$, the out-coupling efficiency is about $54.8 \%(=0.4 \mathrm{~mW} / 0.73 \mathrm{~mW})$.

\section{Conclusion}

We fabricated a novel out-coupling device that has a V-grooved cladding. The theoretical out-coupling efficiency is limited by the maximum core-to-cladding coupling ratio, which is $99.9 \%$ in our device, and the obtained outcoupling efficiency is $54.8 \%$. According to our experience, the out-coupling efficiency can be improved by optimizing the grating and V-grooved cladding. In our device, the optical signal can be directly detected from the fiber without using any lenses, filters, or splitters. Since this device has wavelength-division-multiplexing (WDM) characteristics inherited from the TFBG, the receiver array for WDM signals can be implemented by cascading the device with different grating parameters. The fiber in-line type also makes it possible to develop small-size optical communication devices such as transceivers, receivers, and monitoring devices.

\section{References}

1. J. L. Wagener, T. A. Strasser, J. R. Pedrazzani, J. DeMarco, and D. J. DiGiovanni, "Fiber grating optical spectrum analyzer tap," Eur. Conf. Opt. Commun. 5, 65-68 (1997).

2. S. Wielandy and S. C. Dunn, "Tilted superstructure fiber grating used as a Fourier-transform spectrometer," Opt. Lett. 29(14), 1614-1616 (2004).

3. K. S. Feder, P. S. Westbrook, J. Ging, P. I. Reyes, and G. E. Carver, "In-fiber spectrometer using tilted fiber gratings," IEEE Photonics Technol. Lett. 15(7), 933-935 (2003).

4. Q. Li, C.-H. Lin, A. A. Au, and H. P. Lee, "Compact all-fiber on-line power monitor via core-to-cladding mode coupling," Electron. Lett. 38(18), 1013-1015 (2002).

5. I. Riant, C. Muller, T. Lopez, V. Croz, and P. Sansonetti, "New and efficient technique for suppressing the peaks induced by discrete cladding mode coupling in fiber slanted Bragg grating spectrum," Opt. Fiber Commun. 1, 118-120 (2000).

6. S. Yoon, S. Lee, H. Kang, J. Jeong, C. Yu, and B. Kim, "Highly efficient, wavelength-selective out-coupling of cladding mode using tilted fiber grating and micro-etched cladding," Opt. Fiber Commun., OFP6 (2006).

7. T. Erdogan and J. E. Sipe, "Tilted fiber phase gratings," J. Opt. Soc. Am. A 13(2), 296-313 (1996).

8. T. Erdogan, "Fiber grating spectra," J. Lightwave Technol. 15(8), 1277-1294 (1997).

9. L. Dong, B. Ortega, and L. Reekie, "Coupling characteristics of cladding modes in tilted optical fiber Bragg gratings," Appl. Opt. 37(22), 5099-5105 (1998).

10. K. S. Lee and T. Erdogan, "Fiber mode coupling in transmissive and reflective tilted fiber gratings," Appl. Opt. 39(9), 1394-1404 (2000).

11. C. Jauregui, A. Quintela, J. Echevarria, O. M. Conde, and J. M. Lopez-Higuera, "Experimental characterization of tilted fiber Bragg gratings," Opt. Fiber Commun. 1, 159-162 (2002). 